\title{
Report on the presence of a toxic substance, dimethyl formamide, in sodium fluorescein used for fluorescein angiography
}

\author{
J. S. H. JACOB,' E. S. ROSEN, ${ }^{2}$ AND E. YOUNG ${ }^{2}$ \\ From 'Manchester University Medical School and ${ }^{2}$ Manchester Royal Eye Hospital
}

SUMMARY The revelation that intravenous sodium fluorescein is not all that it might seem to be may be a significant finding in the light of the adverse reactions to fluorescein that have been previously reported. Analysis of commercially prepared intravenous sodium fluorescein by mass spectroscopy and nuclear magnetic resonance has indicated that an industrial solvent used in the manufacturing process has not been eliminated. Dimethyl formamide is an industrial solvent with a maximum acceptable exposure level of 10 parts per million for dermal contact. It has been found in quantities of 5000 parts per million in the fluorescein for intravenous use. This investigation was prompted by a significant increase in the adverse reactions in patients receiving intravenous fluorescein in the retinal photographic unit at the Manchester Royal Eye Hospital.

Fluorescein, [spiro (isobenzofuran-1 $(3 \mathrm{H}), 9^{\prime}$ $-(9 \mathrm{H})=$ xanthem -3 - one $-3^{\prime} 6^{\prime}$ dihydroxy. is made by heating phthaleic anhydride with resorcinol. It is a yellowish red powder which is soluble in water. alcohol. glycerol. dimethyl formamide, and $85 \%$ formic acid. but is insoluble or very sparingly soluble in chloroform, ethyl ether, benzene, and xylene. The sodium salt, molecular weight 376.27 , is freely soluble in water and gives an intense yellowish green fluorescence in blue or ultraviolet light down to a concentration of $0 \cdot 02 \mathrm{ppm} .{ }^{\prime}$

An aqueous solution of sodium fluorescein given intravenously now forms a major part of the assessment of many retinal disorders. $5 \mathrm{ml}$ of $10 \%$ or 3 $\mathrm{ml}$ of $20 \%$ or $25 \%$ solution is injected rapidly intravenously. It is adsorbed on to albumin and other plasma proteins in the blood stream and thus circulates to the eye. The fluorescein is excited within the eye by means of a blue illuminating light, and a photographic record of its passage through the eye is obtained through a yellow-green barrier filter.

Excretion of fluorescein from the body is in urine in a mainly unchanged form, but in part it is conjugated and eliminated by the liver.

Adverse side effects of intravenous fluorescein injection are well documented and include nausea, vomiting, and urticaria as well as some more severe Correspondence to Mr E. S. Rosen. FRCS. 10 St John Street. Manchester M3 4DY. 'anaphylactoid' reactions of laryngeal oedema or even shock and cardiac arrest. The overall incidence of side effects is 10 to $20 \%$ for nausea, 0.1 to $1.4 \%$ for urticaria and vomiting, and 0.6 to $1 \%$ for severe reactions. ${ }^{2}$

\section{Materials and methods}

At the Manchester Royal Eye Hospital a record of adverse reactions to intravenous fluorescein administered in retinal photographic investigations has been routine. Concern was raised recently when an increase from $6.8 \%$ for January to July 1980 to $12.7 \%$ for January to July 1981 was noted in the total number of adverse reactions (Table 1). At the same time the severity of the reactions had increased. These have in order of severity consisted of one case of acute 'anaphylactoid' shock, effects on the central nervous system resulting in one case of temporary deafness, complaints of back pains, and several cases of nerve pains in the arms. In addition there was a marked increase in the frequency of nausea and the severity of the vomiting.

The technique of performing fluorescein angiography had not changed; however, a different batch of fluorescein was being used. Consequently a chemical analysis of the fluorescein was performed to see if an explanation could be found for the increase in the adverse reactions. 
Table 1 Adverse reactions to $3 \mathrm{ml}$ of $25 \%$ fluorescein intravenously

\begin{tabular}{|c|c|c|c|c|c|c|}
\hline & \multicolumn{3}{|l|}{1980} & \multicolumn{3}{|l|}{1981} \\
\hline & $\begin{array}{l}\text { Total no. of } \\
\text { fluorescein } \\
\text { angiograms }\end{array}$ & $\begin{array}{l}\text { No. of } \\
\text { reactions }\end{array}$ & $\%$ Reactions & $\begin{array}{l}\text { Total no. of } \\
\text { fluorescein } \\
\text { angiograms }\end{array}$ & $\begin{array}{l}\text { No. of } \\
\text { reactions }\end{array}$ & \% Reactions \\
\hline January & 118 & 7 & 5.9 & 154 & 23 & 14.9 \\
\hline February & 127 & 10 & $7 \cdot 8$ & 150 & 17 & $11 \cdot 3$ \\
\hline March & 130 & 3 & $2 \cdot 3$ & 149 & 17 & $11 \cdot 4$ \\
\hline April & 102 & 14 & $13 \cdot 7$ & 134 & 17 & $12 \cdot 7$ \\
\hline May & 62 & 5 & $8 \cdot 1$ & 91 & 16 & $17 \cdot 6$ \\
\hline June & 145 & 9 & $6 \cdot 2$ & 68 & 13 & $19 \cdot 1$ \\
\hline July & 163 & 10 & $6 \cdot 1$ & 109 & 10 & $9 \cdot 2$ \\
\hline
\end{tabular}

This analysis revealed the presence of a significant amount of an industrial solvent, dimethyl formamide (DMF), in the fluorescein. The DMF was detected by mass spectrometry and measured by nuclear magnetic resonance spectroscopy. Two samples of $25 \%$ sodium fluorescein showed $11 \%$ and $8.5 \%$ molar DMF with respect to fluorescein. A sample of $20 \%$ sodium fluorescein from the same manufacturing source showed a similar percentage of DMF. Two samples of $25 \%$ sodium fluorescein from a different source made up in our own hospital pharmacy showed no evidence of DMF.

\section{Discussion}

DMF is used in the purification to pharmaceutical standards of crude sodium fluorescein, and it would appear that the incomplete removal of the solvent from the finished powder was responsible for the adverse reactions. DMF is a substance readily absorbed following inhalation or skin contact, and is not intended for intravenous infusion. At lower levels of exposure to DMF by inhalation, direct contact or ingestion, nausea, and vomiting may result. Neurotoxicity, abdominal pain, hepatomegaly, hepatic necrosis, and kidney damage are seen in higher levels of exposure. ${ }^{3}$

Attempts to find a cause for the adverse reactions to fluorescein in the past have also pointed to impurities in the sodium fluorescein. Yannuzzi et al. ${ }^{4}$ found following chromatographic analysis that the presence of impurities increased the incidence of commonly reported side effects of fluorescein. The presence of small amounts of DMF, however, is not readily detected by chromatographic analysis, the use of sophisticated columns being required. Nor is DMF identifiable by fluorescent analytical techniques.
Other workers ${ }^{2}$ attempted to find an allergic cause for the reactions but with limited success.

Further work would have to be done to link DMF directly with the increased number of adverse reactions to fluorescein. However, the presence of DMF, a toxic contaminant in the fluorescein used in the January to July 1981 batches cannot be disputed. Since reverting to the use of purified $25 \%$ sodium fluorescein with confirmed absence of DMF, a sequence of 256 cases has shown none of the violent adverse reactions previously experienced with the contaminated batch. The incidence of nausea and vomiting reactions reverting to around 5\%.

Following notification of the DHSS, users of fluorescein have been circularised warning of the content of DMF in certain batches of the dye. Regulations controlling the permissible exposure to DMF are at present under review. Subsequent to our discovery DMF in fluorescein for intravenous use has been eliminated by obtaining correctly purified sodium fluorescein.

The authors express their sincere thanks to Messrs ICI (Organics) for their invaluable assistance in the analysis of the various samples of sodium fluorescein and to Sister A. Goodfellow and Mrs G. Shawcross of the Retinal Medical Service at the Manchester Royal Eye Hospital for providing statistics and samples from which the authors were able to carry out their investigation.

\section{References}

1 Merk Index. 9th ed. New Jersey: Merk, 3233.

2 Stein MR. Parker CW. Reaction following intravenous fluorescein. Am J Ophthalmol 1971; 72: 861-8.

3 Baselt RC. Biological Monitoring for Industrial Chemicals. California: Biomedical Publications. 1980: 120-1.

4 Yannuzzi LA. Justice J. Baldwin HA. Effective differences in the formulation of intravenous fluorescein and related side effects. $\mathrm{Am}$ J Ophthalmol 1974; 78: 217-21. 